\title{
Honeytubes: Hollow lattice truss reinforced honeycombs for crushing protection
}

\author{
Sha Yin ${ }^{\mathrm{a}, \mathrm{b}, \mathrm{e}}$, Jiani Li ${ }^{\mathrm{a}, \mathrm{b}}$, Binghe Liu ${ }^{\mathrm{a}, \mathrm{b}}$, Kangpei Meng ${ }^{\mathrm{b}}$, Yong Huan ${ }^{\mathrm{c}}$, Steven R. Nutt ${ }^{\mathrm{d}}$, Jun Xu ${ }^{\mathrm{a}, \mathrm{b}, \mathrm{e}, *}$ \\ ${ }^{a}$ Department of Automotive Engineering, School of Transportation Science and Engineering, Beihang University, Beijing 100191, China \\ ${ }^{\mathrm{b}}$ Advanced Vehicle Research Center (AVRC), Beihang University, Beijing 100191, China

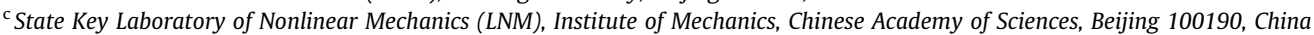 \\ ${ }^{\mathrm{d}}$ M.C. Gill Composites Center, University of Southern California, Los Angeles, CA 90089-0241, USA \\ ' State Key Laboratory for Strength and Vibration of Mechanical Structures, School of Aerospace Engineering, Xi'an Jiaotong University, Xi'an 710049, China
}

\section{A R T I C L E I N F O}

\section{Article history:}

Received 13 October 2016

Revised 5 November 2016

Accepted 5 November 2016

Available online 8 November 2016

\section{Keywords:}

Honeycombs

Lattice structure

Composites

3D printing

Crashworthiness

\begin{abstract}
A B S T R A C T
Lattice truss reinforced honeycombs (LTRHs), termed honeytubes, were developed based on a hybrid design of micro-lattice truss and square honeycomb topologies. Carbon fiber reinforced composite and polymer LTRHs were fabricated using different manufacturing approaches. Out-of-plane compression tests were performed on the LTRHs, and the properties were compared with the conventional square honeycombs. The stiffness and strength values of composite LTRHs didn't surpass those of composite square honeycombs due to the manually induced defects. On the other hand, polymeric LTRHs with perfect geometries were stiffer and stronger than the corresponding polymeric square honeycombs. A parametric study of the buckling resistance was carried out via finite element analysis, and the results indicated that hollow lattice stiffens honeycombs and increases the resistance to buckling, while the specific properties of honeytubes depend on their geometrical parameters. Moreover, the crush force efficiency and specific energy absorption were greater than those of square honeycombs and hollow lattice. This work demonstrates that hybrid designs that capitalize on micro-topologies can populate vacant regions in mechanical property charts, and provide increased energy absorption as crushing protection structures.
\end{abstract}

(c) 2016 Elsevier Ltd. All rights reserved.

\section{Introduction}

The emergence of new engineering materials is often driven by a need to fill gaps in material-property charts to enable new designs [1]. The processes by which these materials are developed includes designing new alloys or polymers based on chemical knowledge, controlling microstructures by thermomechanical processing, and designing hybrid materials that combine materials and/or space in new configurations [2,3]. During the last decade, the development of lattice materials has relied primarily on the latter approach. Lattice materials are periodic cellular materials characterized by efficient micro-architectures and inherent open space, attributes that impart superior mechanical efficiency as sandwich core materials and mechanical metamaterials [4]. Metallic lattice materials have been studied, and their specific properties and energy absorption capabilities were examined and compared with

\footnotetext{
* Corresponding author at: Department of Automotive Engineering, School of Transportation Science and Engineering, Beihang University, Beijing 100191, China. E-mail address: junxu@buaa.edu.cn (J. Xu).
}

traditional sandwich core materials [5-9]. Specifically, hollow metallic microlattice materials based on advanced microfabrication techniques exhibited promising potential for impact protection [10-18]. Additionally, lattice composites, which are hybrids of lattice structures and fiber composites, can yield superior specific properties and populate vacant spaces in low-density regions of materials property charts [19-24]. Recent efforts have focused on fabrication techniques for these lattice composites, including interlocking [25], weaving [26], and hot press molding $[27,28]$, although low-cost and large-volume approaches are still required to bring such structures into use.

Honeycombs, a cellular material widely used in engineering structures, are perhaps the most successful biomimetic material. The most common topologies include hexagonal honeycombs [29], triangular honeycombs [30], square honeycombs [31] and chiral honeycombs [32]. Various mass-production fabrication techniques have been employed to produce such honeycombs at different length scales, as summarized in recent literature [33]. However, because of the extremely low densities, the honeycomb 
cell walls buckle easily in compression, which limits both strength and out-of-plane energy absorption capacity.

Lattice and honeycomb composites in general behave like britthe materials in compression, in that the loading force decreases sharply after reaching a peak. For cellular materials used in impact loading applications, the peak force, crush force efficiency, and energy absorption capacity per unit mass are critical indicators for impact resistance [34-36]. In the present study, we describe the design of hybrid structures that combine the microstructures of hollow lattices [20] and honeycombs [31] to form hollow lattice truss reinforced honeycombs (LTRHs). Carbon fiber reinforced composite LTRHs and polymer LTRHs were fabricated and the corresponding out-of-plane compression tests were carried out, respectively. The buckling resistance and energy absorption capability of these hybrid structures were analyzed to reveal pathways to structures with superior characteristics.

\section{Experimental}

\subsection{Geometry}

The honeytubes in the present study consisted of a square honeycomb and a hollow pyramidal lattice, as shown in Fig. 1. The relative density of the honeytubes is defined as the ratio of solid volume to that of the unit cell, given as

$\bar{\rho}=\bar{\rho}_{\text {rib }}+\bar{\rho}_{\text {truss }}=\left(\frac{2 t}{l}-\frac{2 d t}{l^{2} \sin \omega}\right)+\frac{\pi t(2 d-t)}{2 l^{2} \sin \omega}$

where $t$ and $h$ are rib height and thickness; $l$ is the side length of the square honeycomb; $d$ and $\omega$ are the outer diameter and inclination angle of tubes to the vertical plane in the honeycomb. Note that the thickness of tubes in the present study is defined as one half of the ribs, equal to $t / 2$.

\subsection{Fabrication of composite LTRHs}

The technique used to fabricate the composite LTRHs combines fabrication techniques for hollow pyramidal lattices and square honeycombs, as shown in Fig. 2. First, strips of ribs and lattice trusses (tubes in the present study) were fabricated using thermal expansion molding with steel molds and silicon rubber [20]. Carbon fiber fabric prepregs were stacked along the rough surface of a steel mold with semi-cylindrical grooves, silicone rubber was placed above the grooves, then another ply of prepreg was applied, and finally the other half of the steel mold was overlaid to form the assembly, as shown in Fig. 2a. After curing in an oven at $125^{\circ} \mathrm{C}$ for $1.5 \mathrm{~h}$, the assembly was demolded to yield strips (Fig. 2b). Note that multiple strips were fabricated at the same time. Subsequently, the strips were slotted manually as illustrated in Fig. 2c, then joined by slot insertion as shown in Fig. 2d to form the LTRHs.

To assemble the components and secure the nodes, epoxy adhesive was applied to form the lattice (Fig. 3a). In a separate operation, square honeycomb of the same dimensions was fabricated as shown in Fig. 3b. All specimens were bonded with two composite face sheets using adhesive film to form the sandwich structures. Carbon fiber fabric prepreg $(3234 / \mathrm{G} 803)$ of density $\rho_{f}=1550 \mathrm{~kg} / \mathrm{m}^{3}$ was used for the face sheets.

\subsection{Polymer LTRHs by $3 D$ printing}

Polymer LTRHs were produced using stereo lithography (SLA) based technology, which allowed fabrication of various threedimensional lattice structures. Using an ultraviolet laser, the photosensitive resin was scanned and cured layer by layer. Samples of three geometries were designed using CAD software (Solidworks) and subsequently printed, as summarized in Table 1 . The polymer LTRHs are shown in Fig. 3c and exhibited no apparent defects such as those induced by slot machining in composite LTRHs.

\subsection{Compressive response}

Through-thickness compression tests were performed on LTRHs at a loading rate of $0.5 \mathrm{~mm} / \mathrm{min}$ using a screw-driven testing machine (MTS) with load capacity of $100 \mathrm{KN}$. The load was read directly from the machine while the displacement was recorded using a laser extensometer. The corresponding square honeycombs were similarly tested, and results were compared with those from LTRHs.

For composite LTRHs, the measured compressive response was plotted (see Fig. 4a) along with that of the square honeycombs. The nominal stress increased linearly (approximately) with the nominal strain, and reached a peak stress, followed by a sharp drop when a crack originated from the slot and propagated along the ribs, as shown in Fig. $4 \mathrm{~b}$. The crack continued to propagate until reaching the hollow tube, which induced fiber fracture and then stopped. During this time, the nominal stress did not fluctuate much and an extended stress plateau resulted. Compared with composite LTRHs, the peak load of composite square honeycombs was slightly less, and Euler buckling of ribs occurred. The mechanical properties of the hybrid structures were sensitive to intrinsic fabrication defects, particularly the slots. Actually, defects for the slots in the present study are probably induced by the manually operation which initiate crack propagation easily, but defects a)

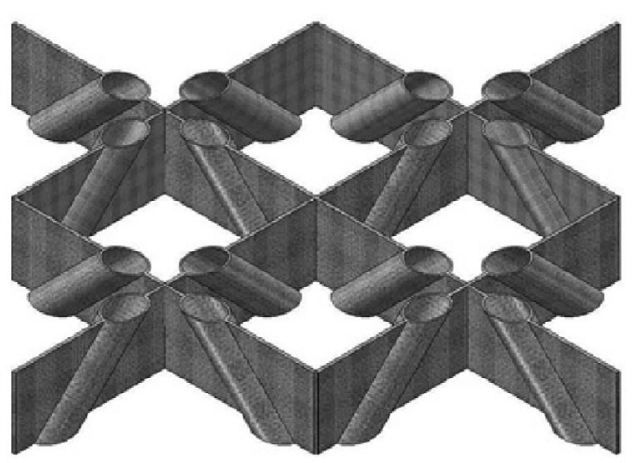

b)

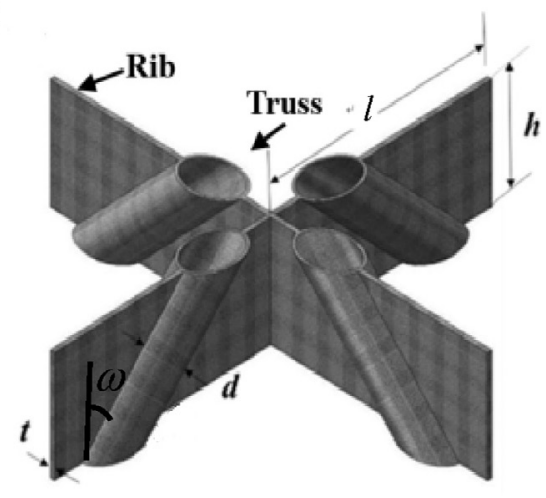

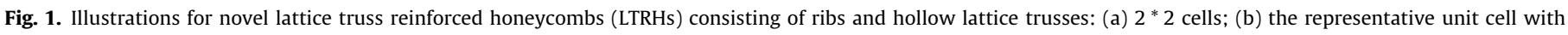
geometrical illustration. 
a)

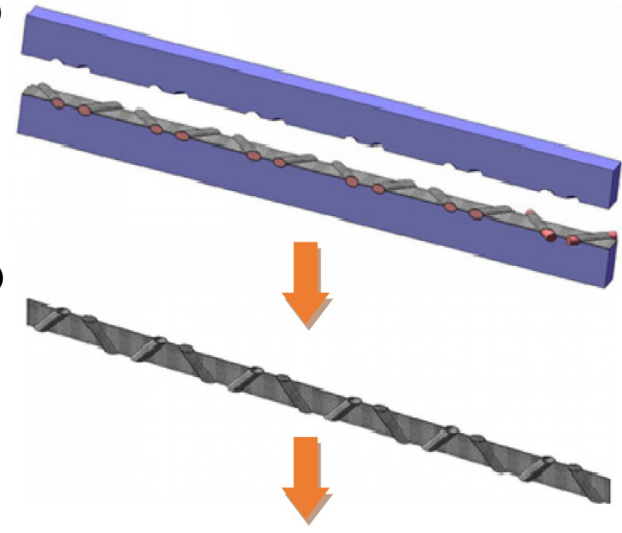

c)

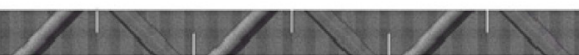

d)

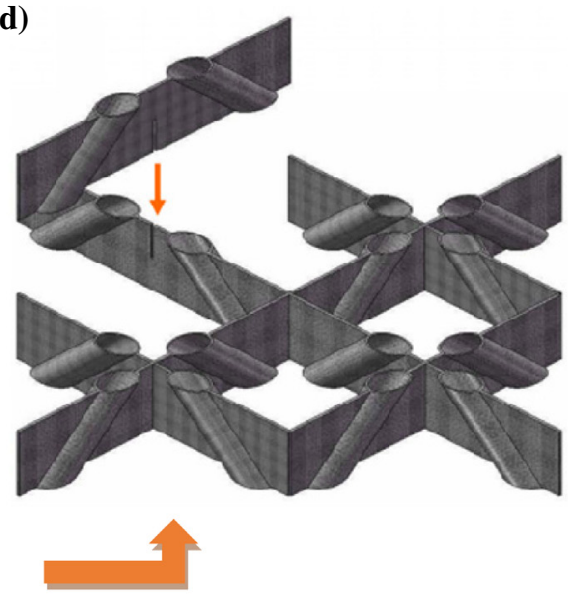

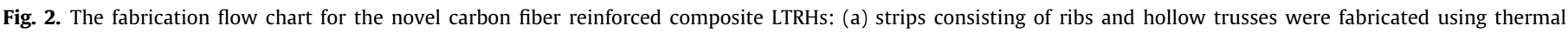
expansion molding; (b) the obtained strip after demolding; (c) arrangement of slots machined in the strip; (d) composite LTRHs by slot insertion.
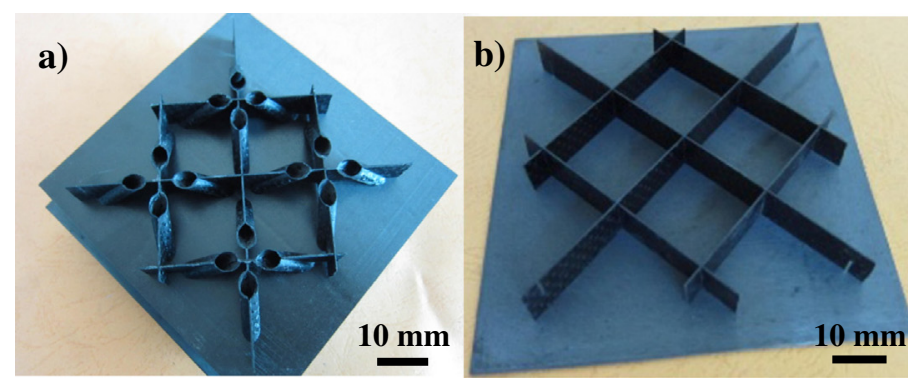

c)

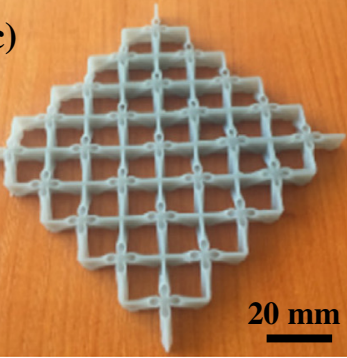

Fig. 3. (a) Carbon fiber reinforced Composite LTRHs and (b) the corresponding composite square honeycombs; (c) polymer LTRHs by 3D printing.

Table 1

Summary of the samples fabricated with different parent materials and geometries.

\begin{tabular}{|c|c|c|c|c|c|c|c|c|}
\hline No. & Material & $h(m m)$ & $l(m m)$ & $t(\mathrm{~mm})$ & $d(m m)$ & $\omega\left(^{\circ}\right)$ & $\bar{\rho}_{r i b}$ & $\bar{\rho}_{\text {truss }}$ \\
\hline 1 & Carbon fiber & 14 & 30 & 0.6 & 6 & 45 & $2.87 \%$ & $1.69 \%$ \\
\hline 2 & Composites & 14 & 30 & 0.6 & 0 & 45 & $4 \%$ & - \\
\hline 3 & Polymer & 7 & 11.885 & 1 & 3 & 45 & $10.82 \%$ & $7.86 \%$ \\
\hline 4 & & 7 & 11.885 & 1 & 0 & 45 & $8.41 \%$ & - \\
\hline
\end{tabular}

a)

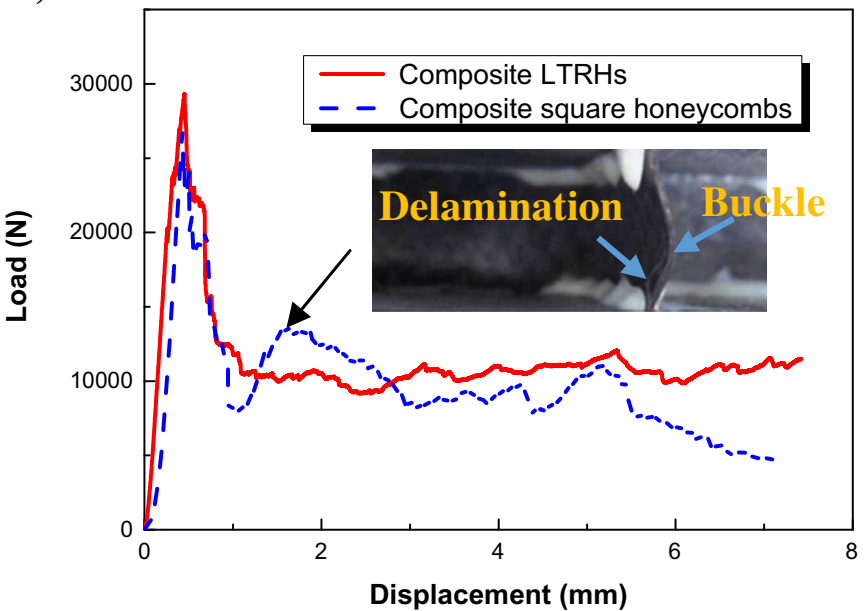

b)

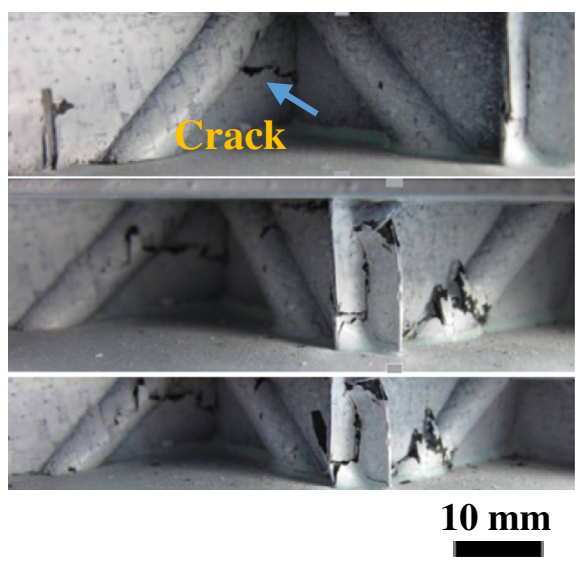

Fig. 4. (a) The compressive behavior of composite LTRHs and square honeycombs; (b) the progressive failure of composite LTRHs. 
may be also affected by the brittle characteristic of carbon fiber composites.

The compressive response and deformation mode of the polymer LTRHs and square honeycombs is shown in Fig. 5. The compressive load increased with displacement followed by an extended plateau (without load decrease) that was accompanied by buckling of rib and tube ends, as shown in Fig. 5b. With continued compressive loading, the structures began to densify and strain harden, resulting in a steep increase in stress. In contrast, compression of the square honeycombs resulted in increasing load followed by decreases when ribs buckled at the onset of densification. The failure modes for structures after densification are shown in Fig. $5 \mathrm{c}$ and d, respectively. The stiffness and strength values of LTRHs were $118 \mathrm{MPa}$ and $4.65 \mathrm{MPa}$, while the corresponding values of square honeycombs were $48 \mathrm{MPa}$ and $3.37 \mathrm{MPa}$, respectively.

\section{Finite element analysis}

Hybrid LTRHs were numerically modeled based on ABAQUS platform to examine parametric effects on buckling behavior and geometric effects on the buckling resistance compared with conventional square honeycombs.

\subsection{Method}

Linear buckling analysis (Eigenvalue buckling) was computationally practical and was used to predict critical loads for structures with ideal geometries. The analysis was carried out to determine the minimum buckling load (the first eigenvalue). All simulations reported were performed on a representative unit cell.
The unit cell of the novel honeycomb was modeled (using SHELL element S4R for $t<2 \mathrm{~mm}$ and SOLID element C3D10 for $t \geqslant 2 \mathrm{~mm}$ following ABAQUS notation). The mesh size was determined to be $\sim 0.5 \mathrm{~mm}$ by convergence check. During the simulation and subsequent analysis, the free sides of the unit cell were simply supported, and all nodes on the top and bottom were fully clamped. The top of the cell was loaded with a unit distribution of forces to create the geometric stiffness matrix under a linear perturbation analysis. The buckling analysis was then performed using a subspace solver with five eigenvalues requested to calculate the fundamental buckling modes.

\subsection{FEA results}

The buckling modes of LTRHs with both slender and stubby trusses are shown in Fig. 6 along with those of square honeycombs. Generally, rib and truss buckling were observed throughout all LTRHs (Fig. 6a and b), while full wave buckling was observed for square honeycombs (Fig. 6c). With periodic boundary conditions, the critical buckling load of LTRHs was much larger than that of square honeycomb, to be discussed in the following section.

\section{Discussion}

Because of the strong constraining effect observed during experiments, we could not treat the mechanical behavior of the hybrid structure (LTRHs) as a simple superposition of square honeycomb and hollow lattice. Hollow trusses incorporated into a square honeycomb inherently exhibit larger buckling loads than ribs of equivalent moment of inertia. We considered the possibility that hollow lattice truss (tubes) acted as stiffeners. LTRHs with dif- a)

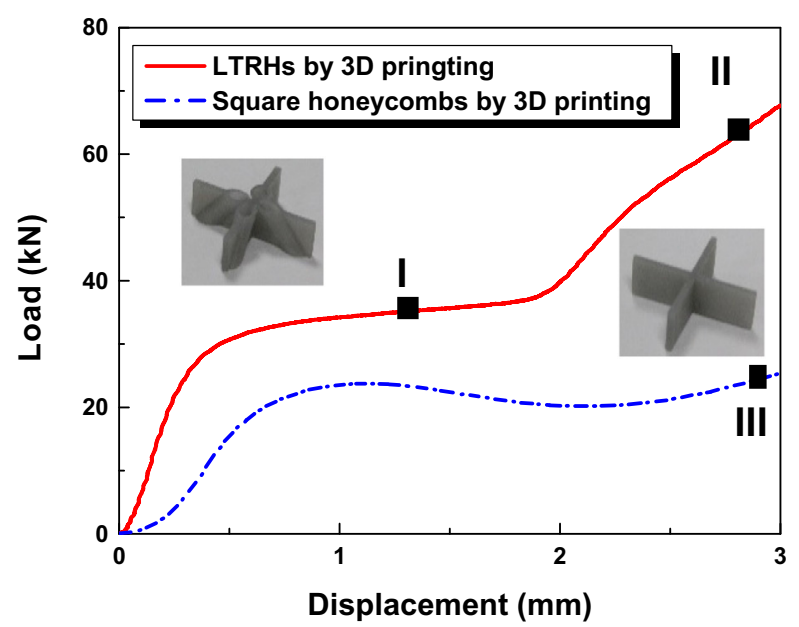

c)

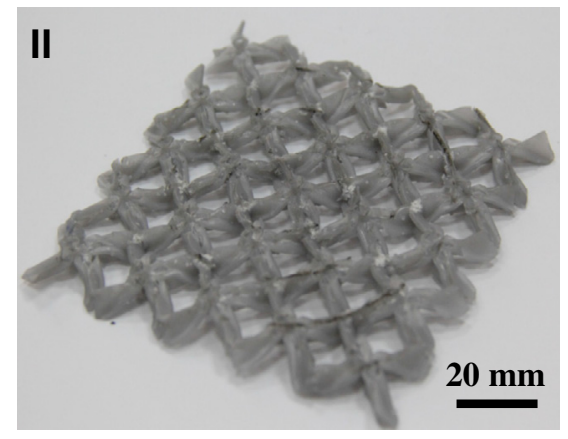

b)

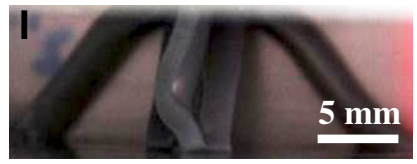

d)

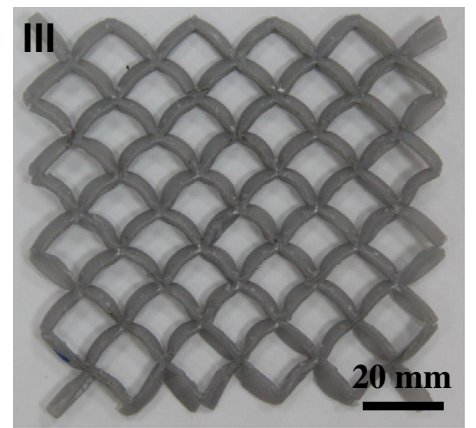

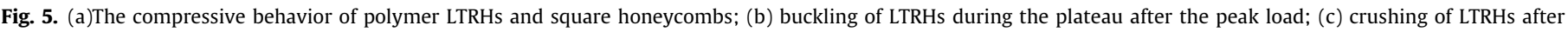
densification; (d) the buckling mode of square honeycombs after densification. 
a)

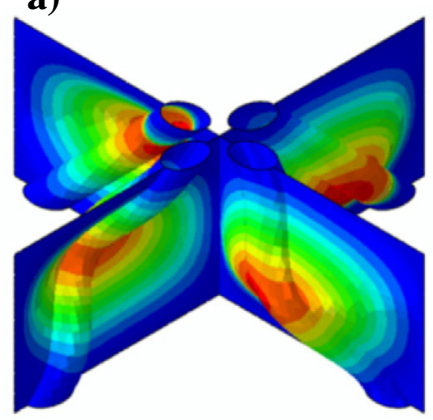

b)

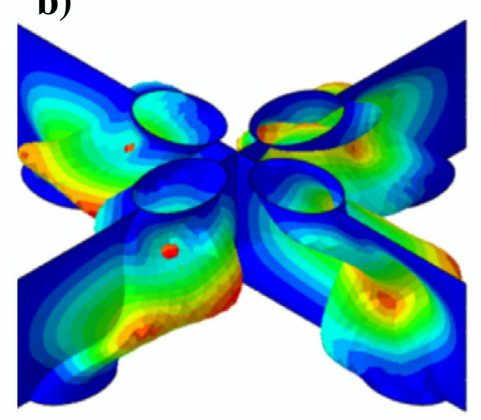

c)

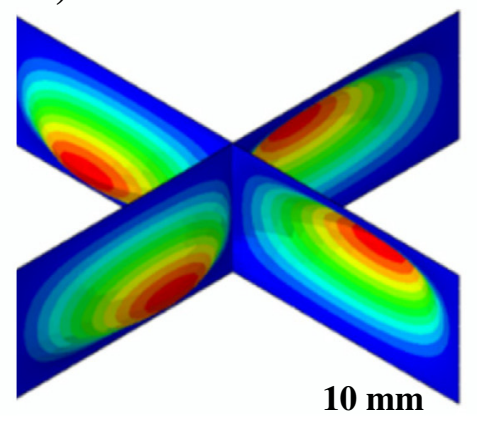

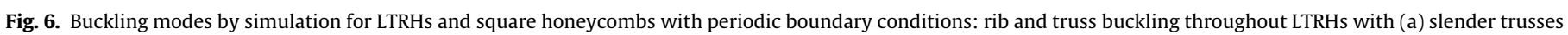
and (b) stubby trusses; (c) full wave buckling of square honeycombs.

ferent normalized geometries $(t / l, d / l, \omega)$ were similarly simulated and compared with the corresponding square honeycombs. In order to analyze the strengthening effect, an index I was defined as the buckling load of LTRHs divided by that of the square honeycombs $F_{L T R H} / F_{S q H}$.

\subsection{Geometrical effects}

The variation of $I$ with different normalized geometries $d / l$ at different values of $t / l$ and $\omega$ is plotted in Fig. 7a. Generally, the buckling load increased after incorporating tubes compared with square honeycombs $(I>1)$. For all values of $t / l$ and $\omega$, the index $I$ increased with $d / l$ first and then decreased, indicating that there was an optimal $d / l$ that provided the greatest strengthening effect. The optimal dimension $d / l$ may be related to the failure mode transition of rib constrained tubes, which is difficult to predict theoretically. Additionally, the strengthening effect is much weaker for larger $t / l$.Fig. $7 \mathrm{~b}$ shows that the index I varies with inclination angle $\omega$ for different $d / l$ and $t / l$. The index increases with the inclination angle for smaller $d / l$, while it decreases with inclination angle for larger $d / l$. Slender tubes of smaller $d / l$ buckle along with ribs. Large deformation of the tube will increase the coupling between the tube and rib, and thus help resist buckling. On the other hand, short tubes of larger $d / l$ may not buckle and may fail by fracture instead. Thus, the coupling effect between tubes and ribs may be weaker and vertical tubes here will contribute to the largest bucking force for the hybrid LTRHs.The weight penalty is introduced after incorporating tubes into square honeycombs. Thus, the strengthening effect considering the relative density variation was also considered. The ratio of specific buckling force $I_{\mathrm{S}}$, termed as $\mathrm{SF}_{L T R H} / \mathrm{SF}_{S q H}$, was compared as shown in Fig. 7c. For thinnerwalled honeytubes, the specific buckling load values are always greater than those of square honeycombs $\left(I_{s}>1\right)$; while for thicker-walled honeytubes, the specific buckling force values may be lower than those of square honeycombs as $d / l$ increases and inclination angle $\omega<45^{\circ}$. In contrast, the buckling resistance of solid lattice trusses are the same order of magnitude as that of ribs, and thus solid lattice truss reinforced honeycomb is not considered here.

\subsection{Crushing force efficiency (CFE)}

In our crashworthiness study, the crushing parameters could be calculated directly from the force-displacement curves. Mean crushing force (MCF) is defined as the average force over a displacement from 0 to densification displacement $d_{D}$ :
$M C F=\frac{1}{d_{D}} \int_{0}^{d_{D}} F \cdot d \delta$

and the crushing force efficiency (CFE) is defined as the ratio of the mean crushing force (MCF) to the peak crushing force (PCF):

$C F E=\frac{M C F}{P C F}$

The crushing force efficiency is a critical index for occupant protection in automotive crush accidents. Unlike most cellular materials, the compressive response after the peak load of LTRHs (with perfect geometry) in compression tests is generally a long plateau with increasing displacement. The duration of the plateau represents larger CFE, a critical characteristic for impact energy absorption [34]. In fact, similar compressive behavior is observed in chiral honeycombs, but was not noted [37]. We attribute the phenomenon observed in this study primarily to the constraint effect between the ribs and cylinders, which also disrupts the natural buckling mode of ribs in square honeycombs.A subsequent simulation using nonlinear explicit formulation was carried out for honeytubes (LTRHs) with different $d / l$ and $\omega$ at $t / l=1 / 15$, and the force-displacement responses are shown in Fig. 8. Note that the material was termed as linear elasticity-perfect plasticity materials for those of woven composites (see experimental section) with compressive modulus of $64 \mathrm{GPa}$ and compressive strength of $557 \mathrm{MPa}$. The data in Fig. 8a shows that the plateau force (or CFE) for honeytube structures is greater than that of the corresponding square honeycombs $(d / l=0)$, and CFE increases with $d / l$ first and then decreases, indicating an optimal value of $d / l=0.2$. On the other hand, Fig. 8b shows the variation of CFE with different inclination angle $\omega$, and honeytube structures with vertical tubes exhibit the highest plateau force and thus the highest CFE.

\subsection{Specific energy absorption (SEA)}

Energy absorption (EA) capacity is defined as the energy dissipated during the crushing process, and determined from the area under the force-displacement curve as

$E A=\int_{0}^{d_{D}} F \cdot d \delta$

where the densification strain, $\varepsilon_{D}=d_{D} / h$, is assumed to be $50 \%$ here. Specific energy absorption is the energy dissipated divided by structural mass $m$ and given by

$S E A=E A / m$. 
a)

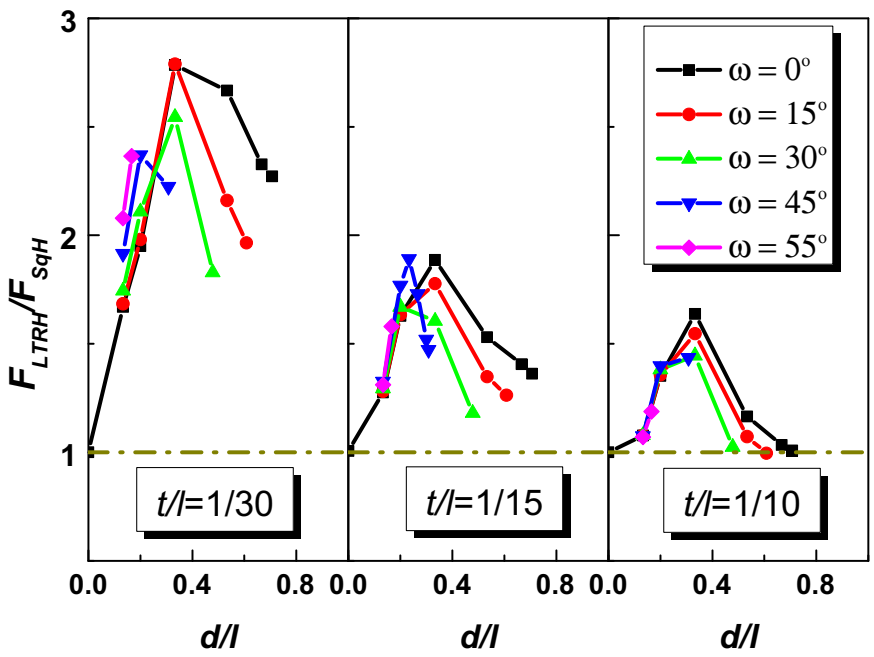

b)

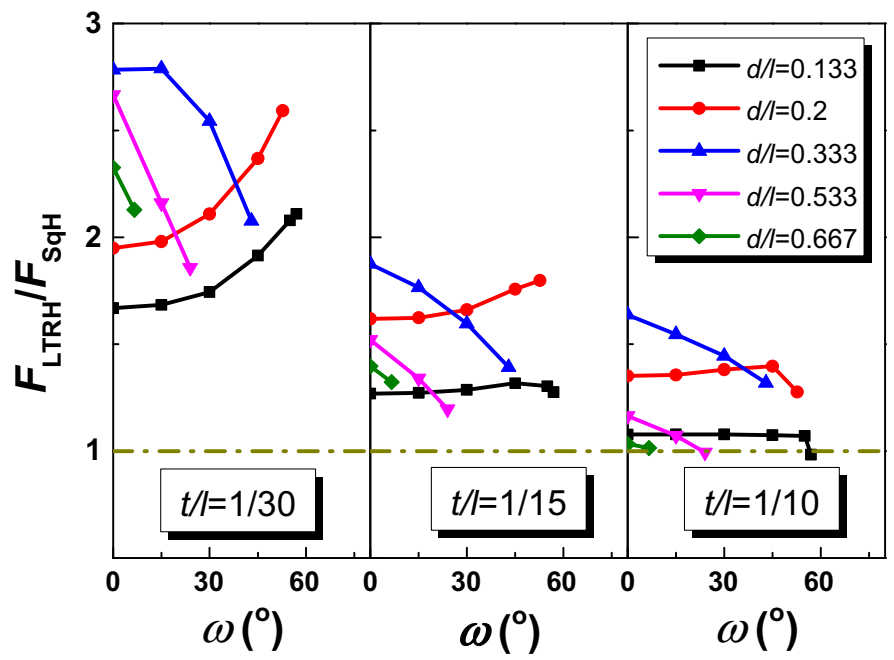

c)

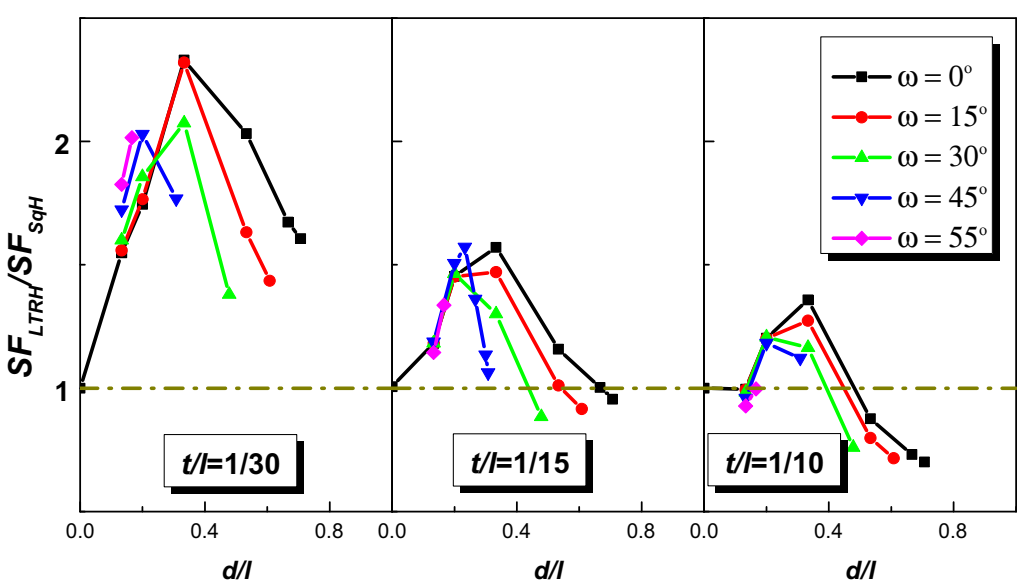

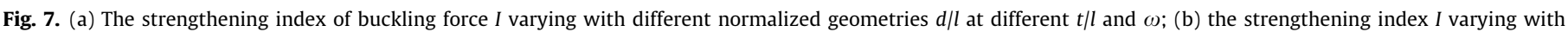

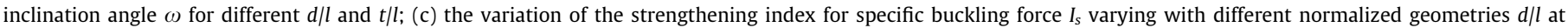
different $t / l$ and $\omega$. Note that $I$ is defined as the buckling load of LTRHs dividing by that of the square honeycombs, while $I_{s}$ is the ratio of specific buckling load.

According to the force-displacement values obtained by simulation, we can calculate the EA and SEA of honeytubes during compression and compare with that of square honeycombs. Similarly, indexes $I_{E A}$ and $I_{S E A}$ are introduced to evaluate the strengthening effect of EA and SEA for honeytube structures compared with those of square honeycombs, respectively. The variations of $I_{E A}$ and $I_{S E A}$ 
a)

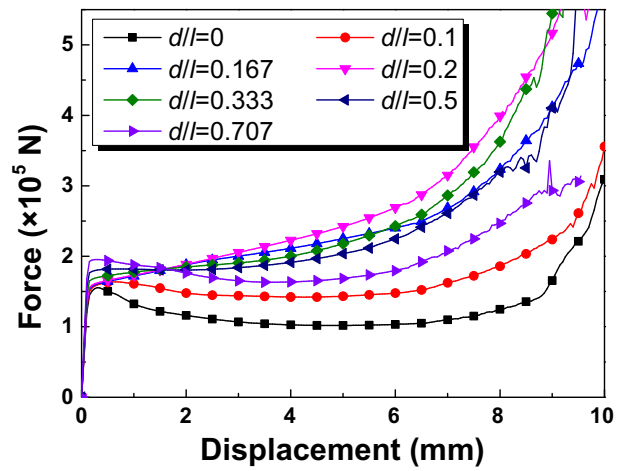

b)

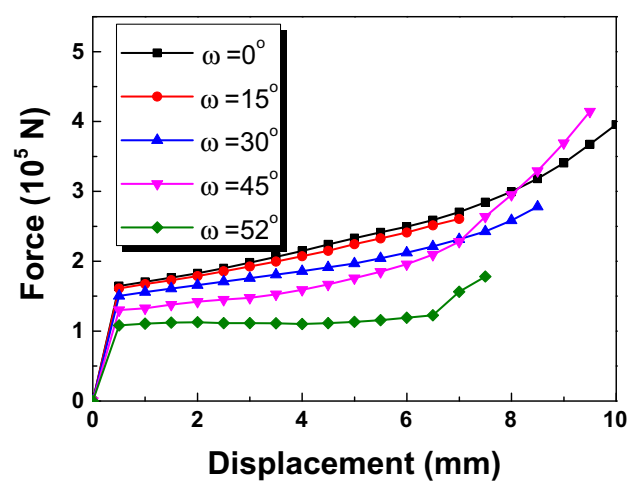

Fig. 8. (a) Force-displacement curves of LTRHs for different $d / l$ with $\omega=0, t / l=1 / 15$ by simulation; (b) force-displacement curves of LTRHs for different $\omega$ with $d / l=0.2$ and $t / l=1 / 15$.

are shown in Fig. 9. For vertical tubes $(\omega=0), I_{E A}$ and $I_{S E A}$ increase with $d / l$ first and then decrease, indicating an optimal value of $d /$ $l=0.2$, while at a constant $d / l$ of $0.2, I_{E A}$ and $I_{S E A}$ decrease with inclination angle. Note that the variation rule here is the same as that of CFE.

\subsection{Comparison with hollow lattice}

The performance of honeytubes was also compared with hollow lattice structures of $d / l=0.2, t / l=1 / 15$ and $\omega=45^{\circ}$ by simulation. The indexes $I, I_{E A}$ and $I_{S E A}$ for honeytubes and square honeycombs were both compared with those of a hollow lattice, as shown in Fig. 10. The buckling force, EA and SEA of honeytube is about 4, 6 and 2.5 times of those of hollow lattice, while for square honeycombs in this case, the buckling force, EA and SEA, are 2.4, 4.5 and 2 times of those of hollow lattice. Thus, the hybrid honeytube structures here outperform both square honeycomb and hollow lattice cores.

\section{Conclusions}

Hollow lattice truss reinforced honeycombs (LTRHs), or honeytubes, were designed to combine lattice topology with square honeycombs. Carbon fiber reinforced composite LTRHs were fabricated by hot pressing and interlocking, while the corresponding polymeric counterparts were obtained by a SLA-based 3D printing. Out-of-plane compressive performance was tested and compared with that of square honeycombs. The stiffness and strength values of composite LTRHs did not surpass those of composite square honeycombs due to the manually induced defects at the slots. However, polymeric LTRHs with perfect geometries were stiffer and stronger than the corresponding polymeric square honey-
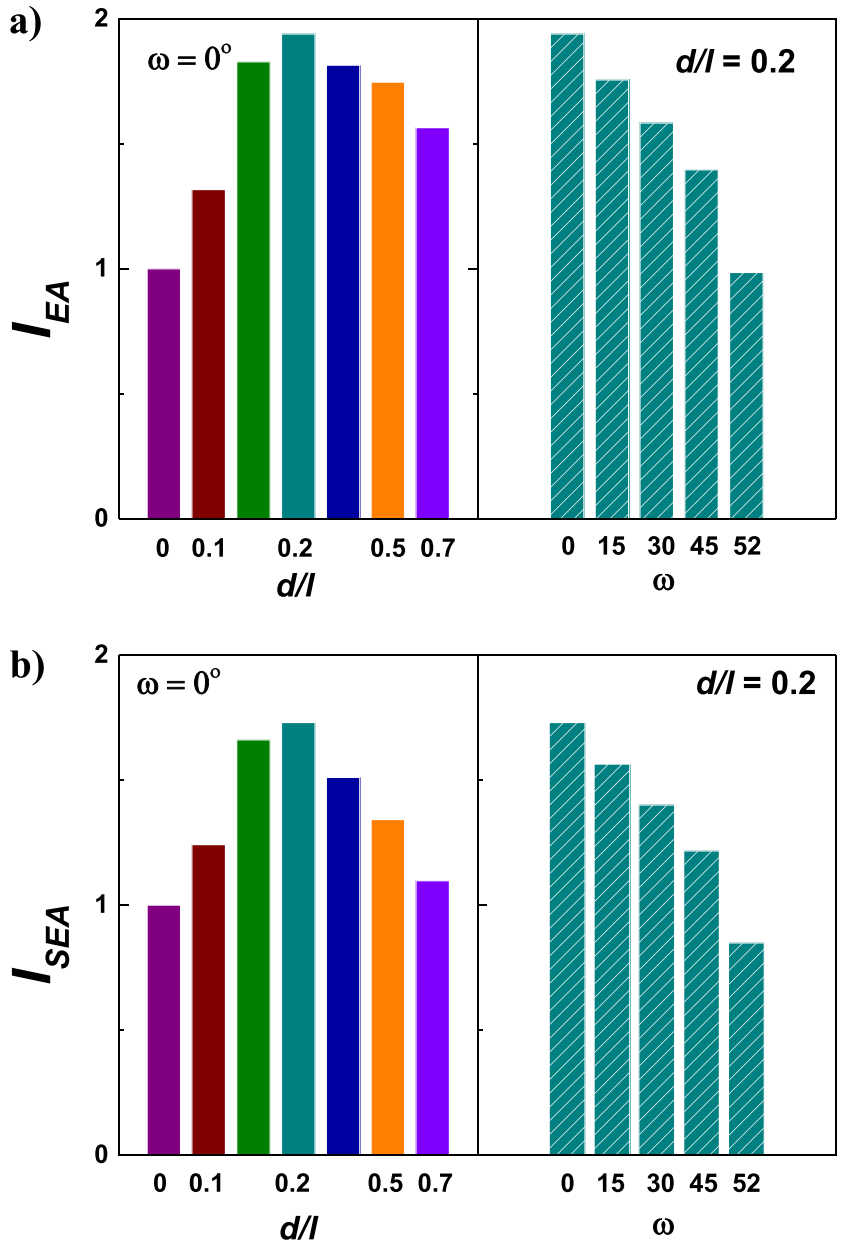

Fig. 9. (a) $I_{E A}$ and (b) $I_{S E A}$ variations with $d / l$ and $\omega$. $I_{E A}$ and $I_{S E A}$ are the strengthening indexes of energy absorption and specific energy absorption for honeytubes (LTRHs) comparing with those of square honeycombs $(d / l=0)$.

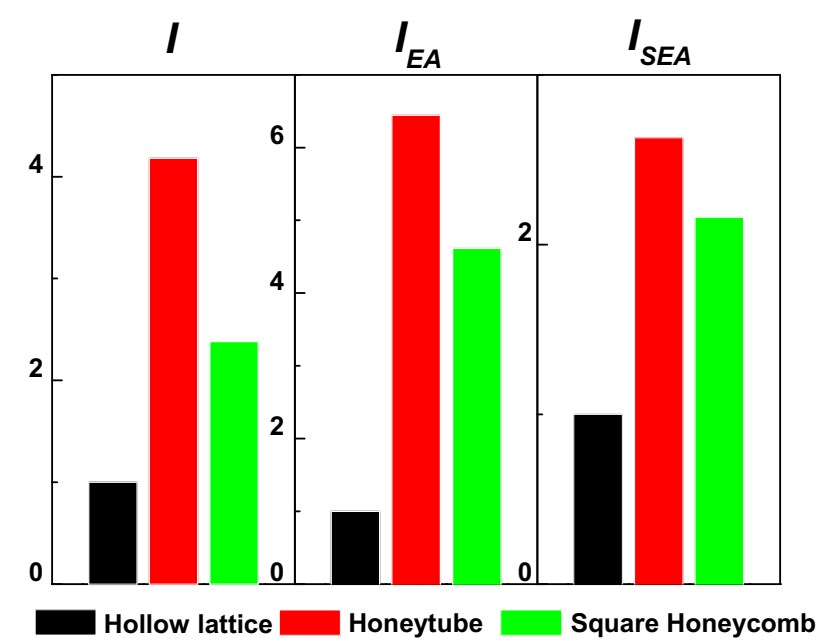

Fig. 10. $I_{E A}$ and $I_{S E A}$ for honeytubes and hollow lattice comparing with square honeycombs with $\omega=45^{\circ}, d / l=0.2, t / l=1 / 15$.

combs. Additionally, the buckling resistance of the novel structures were studied parametrically using FEA. LTRHs with different normalized geometries $(t / l, d / l, \omega)$ were simulated, indicating that the buckling resistance of LTRHs increased after incorporating hollow lattice, while the specific properties of honeytubes depend on 
their geometrical parameters. Moreover, the crush force efficiency (CFE), energy absorption (EA) and specific energy absorption capacity (SEA) of LTRHs were also evaluated and found to be superior to both square honeycombs and hollow lattice structures based on simulation.

The increased buckling resistance and crashworthiness indicate that hybrid designs which capitalize on micro-topologies could be an effective way to create materials that exhibit combinations of properties not possessed by solely materials. Honeytube structures (LTRHs) provide opportunities to vary and optimize energy absorption capacity for crushing protection. In this regard, honeytube structures afford opportunities to expand materials selection options to spaces in materials property charts that are presently vacant. Also, this work demonstrates that hybrid designs could pave a new road for new materials and structures providing better impact and crushing protection. Mechanical performance of honeytube structures at high strain rate will await future research efforts in this vein.

\section{Acknowledgement}

This work was supported by the National Natural Science Foundation of China under Grant Nos. 11402012 and 11372323, the Fundamental Research Funds for the Central Universities, Beihang University, the startup fund for "Zhuoyue 100" titled professor, Beihang University, and Opening fund of State Key Laboratory for Strength and Vibration of Mechanical Structures, Xi'an Jiaotong University.

\section{References}

[1] Fleck NA, Deshpande VS, Ashby MF. Micro-architectured materials: past, present and future. Proc R Soc London A Mat 2010;466:2495-516.

[2] Ashby M. Designing architectured materials. Scr Mater 2013;68:4-7.

[3] Ashby MF, Bréchet YJM. Designing hybrid materials. Acta Mater 2003;51:5801-21.

[4] Evans AG, Hutchinson JW, Fleck NA, Ashby MF, Wadley HNG. The topological design of multifunctional cellular metals. Prog Mater Sci 2001;46:309-27.

[5] Dharmasena K, Queheillalt D, Wadley H, Chen Y, Dudt P, Knight D, et al Dynamic response of a multilayer prismatic structure to impulsive loads incident from water. Int J Impact Eng 2009;36:632-43.

[6] Yungwirth CJ, Wadley HNG, O'Connor JH, Zakraysek AJ, Deshpande VS. Impact response of sandwich plates with a pyramidal lattice core. Int J Impact Eng 2008;35:920-36

[7] Wei Z, Deshpande VS, Evans AG, Dharmasena KP, Queheillalt DT, Wadley HNG, et al. The resistance of metallic plates to localized impulse. J Mech Phys Solids 2008:56:2074-91.

[8] Queheillalt DT, Wadley HNG. Cellular metal lattices with hollow trusses. Acta Mater 2005;53:303-13

[9] Queheillalt DT, Wadley HNG. Pyramidal lattice truss structures with hollow trusses. Mat Sci Eng A-Struct 2005;397:132-7.

[10] Hundley JM, Clough EC, Jacobsen AJ. The low velocity impact response of sandwich panels with lattice core reinforcement. Int J Impact Eng 2015:84:64-77.

[11] Liu Y, Schaedler TA, Jacobsen AJ, Lu W, Qiao Y, Chen X. Quasi-static crush behavior of hollow microtruss filled with NMF liquid. Compos Struct 2014;115:29-40.
[12] Evans AG, He MY, Deshpande VS, Hutchinson JW, Jacobsen AJ, Carter WB. Concepts for enhanced energy absorption using hollow micro-lattices. Int J Impact Eng 2010;37:947-59.

[13] Doty RE, Kolodziejska JA, Jacobsen AJ. Hierarchical polymer microlattice structures. Adv Eng Mater 2012;14:503-7.

[14] Schaedler TA, Jacobsen AJ, Torrents A, Sorensen AE, Lian J, Greer JR, et al. Ultralight metallic microlattices. Science 2011;334:962-5.

[15] Jacobsen AJ, Barvosa-Carter W, Nutt S. Micro-scale truss structures formed from self-propagating photopolymer waveguides. Adv Mater 2007;19:3892-6.

[16] Yin S, Jacobsen AJ, Wu L, Nutt SR. Inertial stabilization of flexible polymer micro-lattice materials. J Mater Sci 2013;48:6558-66.

[17] Liu YL, Wang LF. Enhanced stiffness, strength and energy absorption for cocontinuous composites with liquid filler. Compos Struct 2015;128:274-83.

[18] Liu YL, Schaedler TA, Chen X. Dynamic energy absorption characteristics of hollow microlattice structures. Mech Mater 2014;77:1-13.

[19] Yin S, Wu L, Ma L, Nutt S. Hybrid truss concepts for carbon fiber composite pyramidal lattice structures. Compos Part B-Eng 2012;43:1749-55.

[20] Yin S, Wu L, Ma L, Nutt S. Pyramidal lattice sandwich structures with hollow composite trusses. Compos Struct 2011:93:3104-11.

[21] Wang B, Wu LZ, Ma L, Sun YG, Du SY. Mechanical behavior of the sandwich structures with carbon fiber-reinforced pyramidal lattice truss core. Mater Des 2010;31:2659-63.

[22] Fan HL, Jin FN, Fang DN. Nonlinear mechanical properties of lattice truss materials. Mater Des 2009;30:511-7.

[23] Xu GD, Yang F, Zeng T, Cheng S, Wang ZH. Bending behavior of graded corrugated truss core composite sandwich beams. Compos Struct 2016;138:342-51.

[24] Song ZZ, Cheng S, Zeng T, Yang F, Jing SD, Fang DN. Compressive behavior of C/ $\mathrm{SiC}$ composite sandwich structure with stitched lattice core. Compos Part BEng 2015;69:243-8.

[25] Yin S, Wu L, Nutt S. Stretch-bend-hybrid hierarchical composite pyramida lattice cores. Compos Struct 2013;98:153-9.

[26] Malcom AJ, Aronson MT, Wadley HNG. Three-dimensionally woven glass fiber composite struts: characterization and mechanical response in tension and compression. J Compos Mater 2016;50:25-43.

[27] Xiong J, Vaziri A, Ma L, Papadopoulos J, Wu L. Compression and impact testing of two-layer composite pyramidal-core sandwich panels. Compos Struct 2012;94:793-801

[28] Schneider C, Velea MN, Kazemahvazi S, Zenkert D. Compression properties of novel thermoplastic carbon fibre and poly-ethylene terephthalate fibre composite lattice structures. Mater Des 2015;65:1110-20.

[29] Ajdari A, Jahromi BH, Papadopoulos J, Nayeb-Hashemi H, Vaziri A. Hierarchica honeycombs with tailorable properties. Int J Solids Struct 2012;49:1413-9.

[30] Sun FF, Lai CL, Fan HL. In-plane compression behavior and energy absorption of hierarchical triangular lattice structures. Mater Des 2016;100:280-90.

[31] Cote F, Russell BP, Deshpande VS, Fleck NA. The through-thickness compressive strength of a composite sandwich panel with a hierarchical square honeycomb sandwich core. J Appl Mech-T ASME 2009;76.

[32] Scarpa F, Blain S, Lew T, Perrott D, Ruzzene M, Yates JR. Elastic buckling of hexagonal chiral cell honeycombs. Compos Part A-Appl 2007;38:280-9.

[33] Zhang Q, Yang X, Li P, Huang G, Feng S, Shen C, et al. Bioinspired engineering of honeycomb structure - Using nature to inspire human innovation. Prog Mater Sci 2015;74:332-400.

[34] Lu G, Yu T. Energy absorption of structures and materials. Cambridge, UK: Woodhead Publishing Limited; 2003. p. 385-400.

[35] Hussein RD, Ruan D, Lu GX, Sbarski I. Axial crushing behaviour of honeycombfilled square carbon fibre reinforced plastic (CFRP) tubes. Compos Struc 2016;140:166-79.

[36] Song J, Chen Y, Lu GX. Axial crushing of thin-walled structures with origami patterns. Thin Wall Struct 2012;54:65-71.

[37] Miller W, Smith CW, Scarpa F, Evans KE. Flatwise buckling optimization of hexachiral and tetrachiral honeycombs. Compos Sci Technol 2010;70:1049-56 